\title{
Shock-wave diagnostics of voids and assessment of workload of lining of mine workings based on the construction of cartograms
}

\author{
Anatolii Yalanskyi ${ }^{1}$, and Oleksii Yalanskyi ${ }^{2, *}$ \\ ${ }^{1}$ Institute of Geotechnical Mechanics named by N. Poljakov of National Academy of Sciences of \\ Ukraine, 49005, Dnipro, Simferopolska Str., 2a, Ukraine \\ ${ }^{2}$ National Technical University Dnipro Polytechnic, 49005, Dnipro, Yavornytskoho Ave., 19, Ukraine
}

\begin{abstract}
In the work, the peculiarities of shock-wave diagnostics of voids and the estimation of load of lining of mine workings based on the construction of cartograms are considered. Mapping is the most visible and informative way of presenting measurement results. For mapping, select such areas that provide equal scales along different axes in the image sweep the surface. The image format contains options for automatic contouring, displaying contour lines, and printing of levels of values for the corresponding contours. The mapping operation itself automatically makes it possible to estimate the depth of the cavities being monitored, and this definition is accurate in terms of area and relative depth, since it is based on automatic processing of measurement results. Only refinements are needed to determine the absolute value of the depth by measuring methods at various bases or drilling holes.
\end{abstract}

\section{Introduction}

To ensure the sustainability of mine workings, operational and long-term monitoring of rock outcrops is necessary. Safety regulations provide for periodic monitoring at various levels, mainly based on visual inspection. A great deal of experience has been gained in using the sonic (seismic acoustic) method in mines producing coal, iron ore, uranium, and so on. However, this method does not allow to localize minor stratification, voids, which may not cause a serious accident, but, nevertheless, during operation they represent a serious permanent danger to the life of miners. After drilling and blasting, as a rule, the focus is on the safety of the roof, and the order and frequency of diagnosis are determined by geological conditions. The shock-wave method is based on shock excitation, registration and analysis of the natural oscillations of the studied portion of the rock mass. Special devices are used to excite vibrations in the system, and contact sensors, such as accelerometers or seismic receivers, are used to receive them. The main informative parameters for shock-wave diagnostics are: vibration amplitudes without determining the

*Corresponding author: yalanskiy.o.a@gmail.com 
frequency (peak amplitude); dominant frequency; maximum amplitude of oscillations at a given frequency; the spectral composition of the oscillations actually determined by a discrete set of amplitudes at fixed frequencies; the duration of the oscillatory process or its parts. However, even in general, they do not allow for a visual assessment of the state of the monitored object, both during diagnosis and during repair [1]. The aim of the work is to consider the features of shock-wave diagnostics of voids and load of mine workings based on the construction of cartograms.

\section{Main study content}

\subsection{Physical-technical basis of the method and methodology of control}

The method of shock-wave diagnostics in mine conditions is used mainly to assess the state of natural and artificially created plane-parallel structures of the rock mass or to estimate the bearing capacity of mine workings. It consists in registering the vibration parameters of an object in the region of sound frequencies. The physical feature of the method is as follows. When an array is excited by a shock, the wave energy due to its divergence decreases in proportion to the square of the radius, in addition, the wave attenuates in proportion to the radius. If the reflection coefficient from the adjacent layer is significant, then only waves that are resonant to the width of the structure are recorded on the exposed surface at some distance from the excitation source. The acoustic field of such a uniform normal wave in the layer has the form [2]:

$$
u_{n}=A_{n} \exp \left\{i \omega\left[t-\frac{x}{v}\left(1-\left(\frac{n \lambda}{2 a}\right)^{2}\right)^{\frac{1}{2}}\right]\right\} \sin \left(\frac{\pi n}{a} y\right),
$$

where $u$ is the offset, the function of coordinates and time; $A$ - amplitude, in general, a complex value; $\omega$ - cyclic frequency; $t$ - time; $x, y$ - coordinates; $v$ - speed of elastic waves, $n$ - mode number, $\lambda$ - wavelength, $a$ - waveguide width.

A family of equations that differ in number describes perturbations that do not change their form when propagating in a layer, which is performed under the condition:

$$
\lambda<\lambda_{c r}=\frac{2 a}{n},
$$

where $\lambda_{c r}$ is the critical wavelength for the mode with the number $n$, it corresponds to the critical frequency $f_{c r}=(v n) /(2 a)$. At wavelengths $\lambda<2 a$ or frequencies $f>v /(2 a)$ in the layer, you can excite any of the modes. The mode with the longest wavelength equals 1 . When $\sin \theta=1$ and $\theta=\pi / 2$ the layer turns into a resonator, and a standing wave forms between the walls, the amplitude of which is constant along the layer. If condition (2) is not satisfied, then (1) takes the form [2]:

$$
u_{n}=A_{n} \exp \left\{-\frac{\omega}{v} x\left[\left(\frac{n \lambda}{2 a}\right)^{2}-1\right]^{\frac{1}{2}}\right\} \exp (i \omega t) \sin \left(\frac{\pi n}{a} y\right),
$$

with increasing distance along the layer axis, the wave will decay exponentially.

Thus, areas of the rock massif, bounded on the one hand by workings, and on the other hand by voids, cracks or other rocks, can form classical acoustic waveguides in the surface 
layer. As a result, the divergence and attenuation of elastic waves are eliminated or reduced, therefore, their propagation along the layers occurs with less attenuation than in an unlimited homogeneous or inhomogeneous medium. The only kind of wave propagating in such acoustic waveguides without changing its structure is normal waves (modes). The closure of cracks, delaminations in the massif or between the concrete lining and the massif under the influence of the load unambiguously translates a uniform normal wave into a non-uniform, exponentially decaying wave. At the same time, at a certain distance from the point of excitation, one, two or three relative maxima will form on the spectral characteristic of the wave, the frequencies of which determine the width of the structures and the amplitudes the array stratification [1].

The most convenient method of visual analysis of the stress-strain state is the mapping method. The calculation system allows you to directly enter a one-dimensional vector or a two-dimensional matrix with the number of elements not exceeding one hundred. However, the number of measurement points in one mine workout or tunnel far exceeds this limit. Splitting the source data array into separate groups is undesirable because in the sections for which splitting is performed, edge effects appear when mapping the surface of voids and areas of increased loads, as a result of which individual sections of the map will not fit together well. In this regard, it was decided to write the source data to the hard disk of the computer as separate files and enter them into program using the Read command [3]. The source data file is a sequence of characters divided into lines, each of which contains data about measurements performed on a single section of the mine or tunnel. The encoding type is "Text" or "Windows Text". Numbers corresponding to individual dimensions are separated by Tab. Measurement data on the cross-sections of development are separated by the symbol of the end of the line "End of Line. EOL". The end of the source data file is indicated by the corresponding symbol "End of File. EOF". The source data file is saved on the hard disk of the personal computer with the format designation "File name" or "File name.extension". The read statement must contain the full path to the file or only the file name if it is located in the working directory of the program shell [3].

In Figure 1 shows a map of the voids around the walkway of the Coal Mine A.F. Zasiadko. This map shows the influence of the deterministic component on the stresses resulting from the extraction of previous lavas.

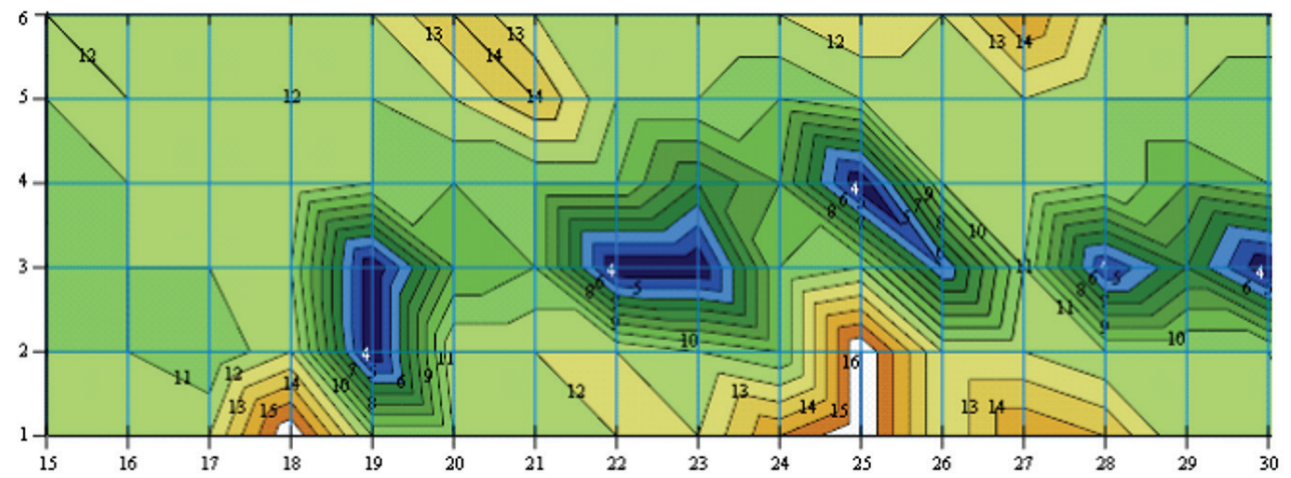

Fig. 1. Distribution maps of areas of increased load lining (dark areas) and voids behind the lining (light areas).

At ore deposits, the main objects of control are mining, carried out by drilling and blasting in rocks of medium strength or very strong, but characterized by an increased number of cracks. The depth of their development is from 300 to $700 \mathrm{~m}$, and the height of the workings is from 2.5 to $4.0 \mathrm{~m}$. The coefficient of strength of rocks of average strength is within 8 - 12, and for strong (granites and iron-containing ores) 19 - 30, irregularities reach 
$0.5 \mathrm{~m}$. Cracks are easily identified, since in a closed space the damping of elastic waves decreases sharply.

Underground mining of valuable ores has always been a problem for mining science and practice. For example, the essence of uranium mining processes differs from the mining of most other minerals by the higher complexity of technological cycles. The dependence of production on mining and geological conditions, the lack of technical capabilities for their accurate prediction introduce elements of uncertainty and unpredictability, complicating the choice of rational technologies. Due to the genetic conditions of uranium accumulation, ores have numerous inclusions of rocks and low-quality ores, layers that often break, pinch out and reappear, break up into separate bodies, which leads to significant losses and depletion of ore. The development of such ores is associated with the need to have a developed network of mine workings, which significantly affects the state of the rocks. In addition, most of the deposits are under water. Artificially, but forcibly, conditions are created for the possible unloading of the array into the void, when the stressstrain state reaches values that can cause dynamic manifestations of rock pressure in the form of impacts. The main feature of these dynamic manifestations is that they are closely related to the explosions and occur during or after the explosions. For an unprotected surface, the main anomalous areas with an increased signal amplitude are peeling. Low oscillation amplitude is observed in the stress zones of the array.

The depth of control is determined by the impact force, the sensitivity and resolution of the indicator, the distance between the points of excitation and the reception of oscillations. The base of control must be at least twice the size of the controlled area. Impact power is regulated in a simple way using devices of different masses. Rationalizing the impact force using a spring as an energy storage device was inefficient due to the low control speed. Accounting for the impact force reduces the usability of the device due to the presence of additional wire. The nature of the reaction to impact is determined by resonance phenomena in block or plane-parallel structures of the controlled zone. Defects of the structure, in particular, inside the layer or at the interface of layers with different properties, cracks simultaneously lead to an increase in the amplitude of free oscillations, which corresponds to the appearance of pronounced resonances in the spectrum and an increase in attenuation in the medium during propagation along a uniform layer. Reducing the connection between the layers of laminated material increases the amplitude of free vibrations, and also shifts the maximum spectral density towards lower frequencies. Solid, durable material of sufficient rigidity reacts to impact with a relatively high frequency, but the transition of the oscillatory process into a wave is the fastest process of energy transfer and, as a result, leads to a rapid damping of the oscillations.

To determine the dimensions of voids by their thickness (depth), measurements are necessary, primarily at different test bases and in different directions, Figure 2 . In the repaired areas, the technological complex was used to diagnose the quality of repair and construction work, which determines the heterogeneity of the concrete shell and the degree of its connection with the massif. To increase image clarity, all maximum levels of oscillations are limited to the 16th level, which is equivalent to $42 \mathrm{~dB}$. Such a restriction in this case does not lead to the loss of information, since there are few large expanded voids, they clearly stand out against the general background. Since it is difficult to estimate the size of voids with a constant base of control; therefore, a maximum level limit was introduced that allows a detailed study of the load along the tunnel with a large number of gradations in areas of average load.

\subsection{Control equipment}

The design characteristics of devices are largely determined by the methods of excitation 
and reception of vibrations.

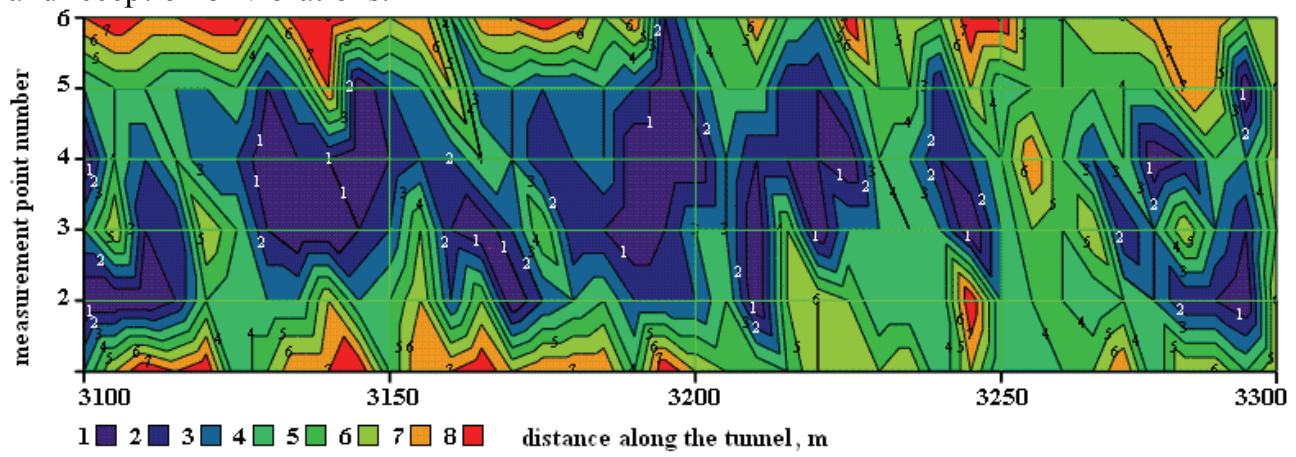

a)

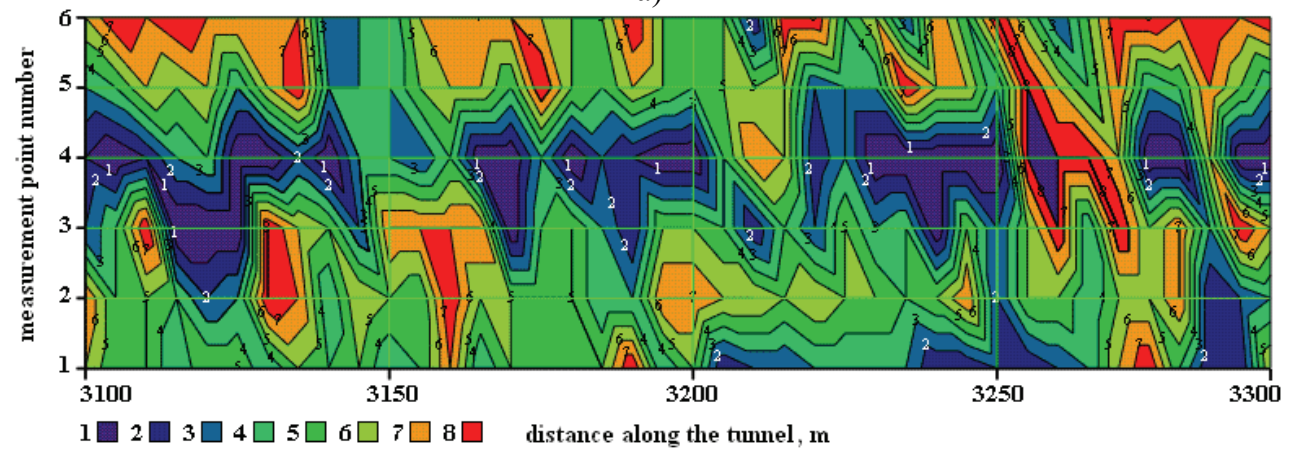

b)

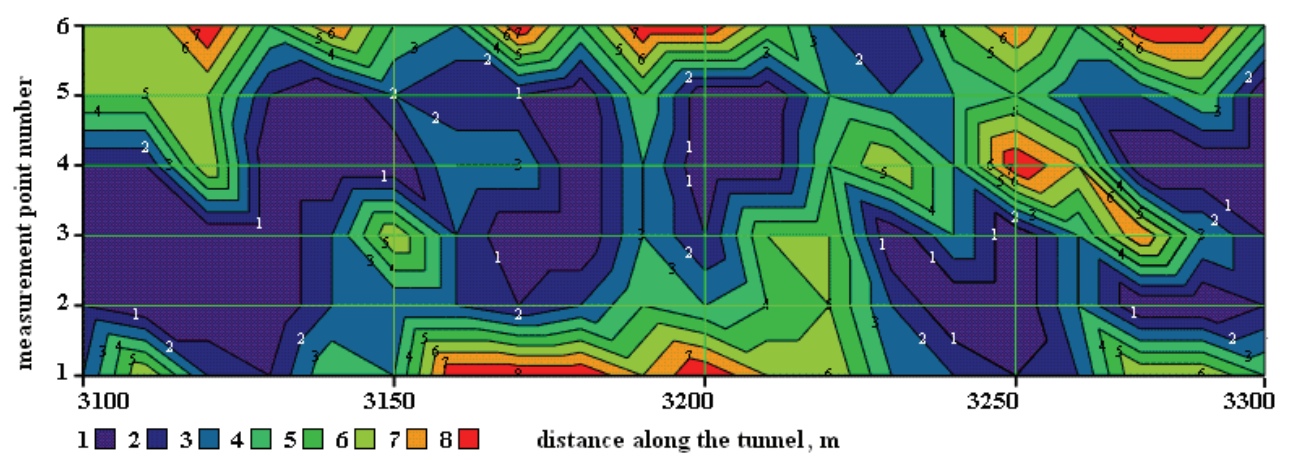

c)

Fig. 2. Map of voids around the lining of the section of the tunnel number 1 of the channel "DniproDonbas", which confirms the modal structure of plane-parallel structures: a - base of control along the tunnel - $5 \mathrm{~m}$, mass of a drummer - $1 \mathrm{~kg}$; $\mathrm{b}$ - base of control on the lining ring - about $5 \mathrm{~m}$, drummer mass $-1 \mathrm{~kg}$; $\mathrm{c}$ - base of control along the tunnel $-10 \mathrm{~m}$, mass of a drummer $-2 \mathrm{~kg}$.

Operating experience of existing developments has shown that their disadvantages are cable connections between the drummer and the electronic unit, as well as the receiver and the same unit of the device. The development of purely contactless communication options has not yet yielded positive results. As the main means of control, the roof condition indicator ISK-1Sh (normal or explosion-proof) is used, which is distinguished by high efficiency and informativeness. This is an octave (from $32 \mathrm{~Hz}$ to $16 \mathrm{kHz}$ ) spectrum analyzer of acoustic and electrical signals with a parallel principle of operation, allowing them to be assessed in ten frequency bands in just a few seconds when using one beat. The number of 
measurement levels is nine in one range, the range is three, the difference between the levels is $3 \mathrm{~dB}$. The indicator is battery powered, the time of continuous operation is 20 hours. The indicator device is designed to control visible and hidden beams, bundles and impacts in ore and coal mines, assess the relative stability of various objects of underground and surface construction. The indicator is equipped with a drummer, a seismic receiver on the rod and a connecting cable. As a pathogen, you can use a conventional hammer or drummer of a special design. Synchronization with the moment of impact is not provided. For diagnostics of objects removed at a distance of more than $2 \mathrm{~m}$, the use of a receiver and a drummer on special extension rods is provided. Work in the mine is accompanied by technological noise, in particular acoustic noise, which propagates both in the air and in the array. The most intense sources of interference are perforators, local ventilation fans, and mining vehicles. Unrecoverable noise includes noise caused by the movement of mine waters. There are practically no sources of electromagnetic radiation that can affect electronic equipment. The level of background radiation does not require the use of special protection from exposure to ionizing radiation, but can serve as a basis for additional radiometric monitoring. The temperature in the workings is positive, does not exceed 10 $40{ }^{\circ} \mathrm{C}$, humidity $95-100 \%$. In the mines there are local tributaries of water, so it is possible that water falls on the tools. There are no power sources from the mine source. The equipment can be transported and monitored by one person, however, to ensure the safety of work, the controls are carried out by two operators, and they must be located outside the area of possible rock fall. Given that the operator must reach to any point of the roof, not being directly under it, the length of the rod with a hammer must be at least $2 \mathrm{~m}$. It is advisable to make the bar of such length removable in order to reduce its size during transportation.

\section{Conclusions}

Shock-wave diagnostics determines the presence of voids, the condition and loading of the lining, the size of solid and detached sections. Its implementation is based on the study of the laws of free oscillations of an object or part of an array, excited by a point impact. Taking into account the assigned tasks, the following options for controlling the exposed surface of the rock massif, lining the trunk, workings, tunnels, foundations of underground and above-ground structures were tested.

\section{References}

1. Yalansky, A.A., Yalansky, Alexey A., Arestov, V.V. (2004). Features of the method of acoustic sounding of the deep structure of the rock mass, Geotechnical mechanics, Dnepropetrovsk, 51, 285 - 296

2. Vinogradova, M.B, Rudenko, O.V, Sukhorukov, A.P. (1990). Theory of Waves. Moskva: Nauka

3. Dyakonov, V.P., Abramenkova, I.V. (1999). MathCAD 7.0 in mathematics, physics and in the Internet. Moscow: Knowlick

4. Ikonnikova, N.A., Korsun, V.I., Slashchev, A.I., Yalansky, Alexey A., Yalansky, A.A. (2015). Modeling and control of dynamic processes in problems of assessing the state of geotechnical systems. Dnipropetrovsk: NSU 